PAPER

\title{
Improving perceived elevation accuracy in sound reproduced via a loudspeaker ring by means of equalizing filters and side loudspeaker grouping
}

\author{
Julián Villegas* \\ Computer Arts Laboratory, University of Aizu, \\ Tsuruga, Ikki-machi, Aizu-Wakamatsu, 965-8580 Japan
}

(Received 9 August 2018, Accepted for publication 18 October 2018)

\begin{abstract}
A spatialization method for loudspeakers arranged in a ring layout (like the one specified in the ITU recommendation ITU-R BS.775) is presented. The proposed method can be described as an improvement to amplitude panning methods in which a frequency-dependent gain is applied to mitigate the effect of loudspeaker locations on the desired signal. By grouping side loudspeakers it is possible to display elevated sources, which is difficult to achieve otherwise in single layer loudspeaker arrays. Objective evaluations showed that the proposed method produces less spectral distortion than 2D-VBAP, but degrades to cross-talk cancellation. Experimental results suggest that, despite the differences regarding cross-talk cancellation, the proposed method yields accurate azimuth and elevation estimations of sound sources in anechoic and echoic conditions except when they are located below the ear level.
\end{abstract}

Keywords: Loudspeakers array, Elevation perception, Equalizing filters, Sound spatialization

PACS number: 43.66.Qp, 43.66.Rq [doi:10.1250/ast.40.127]

\section{INTRODUCTION}

According to several sources (UN-ECE, EUROSTAT, etc.), average house sizes vary dramatically with country. Whereas in 2012 Australia and USA residents enjoyed $>76 \mathrm{~m}^{2}$ per house inhabitant, residents of countries like Japan or Hong Kong enjoyed about $15.5 \mathrm{~m}^{2}$. Unrelated to house size, the game industry and improvements in distribution of high definition audiovisual content over the internet have increased the interest in multichannel stereophony, to fully express these media.

The 5.1 loudspeaker layout specified in the recommendation ITU-R BS.775-3 [1] is one of the most widely adopted worldwide. In such a ring array, sound lateralization is relatively easy to achieve, in contrast with sound elevation. Increasing the number of rings and actuators [2] improves elevation expression shortcomings, but this is challenging for small dwellings or budgets. Another alternative is using signal processing approaches such as cross-talk cancellation [3-5], etc. In this article, a method to express elevated audio imagery in a single ring of loudspeakers is investigated. The method uses filters to compensate for the effect of the loudspeaker locations, and

*e-mail: julian@u-aizu.ac.jp grouping of actuators to achieve artificial elevation. The following section reviews relevant spatialization techniques. Then, the proposed method is explained and evaluated objectively and subjectively. Finally, a discussion on the results of these evaluation is offered before conclusions.

\section{BACKGROUND}

Center frequencies of spectral peaks and notches produced by the pinna and upper body reflections depend on the location of a sound source relative to a listener. Relative energy in different spectral bands has been associated with the perception of different directions [6,7]. E.g., the presence of an octave notch with lower cutoff frequency between $4-10 \mathrm{kHz}$ along with an energy peak above $13 \mathrm{kHz}$ has been associated with frontal localization [8]. It follows that these monaural cues are important for sound elevation perception $[9,10]$.

A conceptually similar approach to the one proposed here was made by Chun et al. [11]: elevation was simulated by applying a spectral notch filter along with a directional band reinforcement to a binaural signal fed to a pair of loudspeakers in front of a listener. It was reported that sounds processed that way were perceived on average $>20^{\circ}$ higher than unconditioned equivalent sounds. 
It is possible to simulate elevated sources using loudspeaker arrays by means of High-Order Ambisonics (HOA) and Wave-Field Synthesis (WFS) [12]. However, the number of transducers needed to achieve realistic results yields these techniques impractical for many rooms.

Regarding WFS, it seems more suitable for horizontal than vertical localization as location accuracy of elevated virtual images produced with this technique is inferior to that obtained with other techniques [13]. It seems possible to improve these WFS shortcomings by extracting elevation cues from Head-Related Impulse Responses (HRIRs-or their equivalent in frequency domain called HRTFs) on the mid-sagittal plane and pre-filtering a sound with such cues [14]. This hybrid technique has been extended to multizone reproduction systems as well [15]. In the pre-filtering step, HRTFs at different elevations in the mid-sagittal plane are convolved with the inverse of the HRTF at elevation $\phi=0^{\circ}$ to eliminate the effect of a sound source projected from the front since this effect is already provided by a loudspeaker at that location. This step is similar to one of those described in Sect. 3. The WFS improvements obtained with the introduction of elevation pre-filtering are "not intended to provide accurate localization of virtual sources in elevation, but to give the listeners the possibility of experiencing elevation effects" [14].

In the context of Vector-Base Amplitude Panning (VBAP) [16], a single frequency-independent gain is applied to signals of different loudspeakers in standard VBAP. However, the magnitude spectrum is unequally affected by the direction of a sound source around a listener. Furthermore, 2D-VBAP is not suitable for displaying elevated sources from a single horizontal loudspeaker ring array.

Frequency-dependent gains have been explored in several ways for VBAP: Pulkki proposed a frequencydependent gain to mitigate an observed coloration in amplitude-panned sources and to improve their perceived localization accuracy [17]. He also proposed "Non-unitary VBAP" (NVBAP) [18], a modification of the standard VPAB whereby a frequency-dependent gain is applied to compensate for an observed bias to rate localizations towards the mid-sagittal plane. More recently, Mikko-Ville et al. proposed another refinement of VPAB in which frequencydependent gains are used for loudness equalization across different rooms and reverberations [19]. None of these revisions are intended to allow elevation expression in a ring of loudspeakers.

Displaying elevated virtual sources in a ring of loudspeakers has been explored before by Kim et al. $[4,5,20]$. They took advantage of surround loudspeaker locations proposed by the ITU-R BS775-3 recommendation (at azimuth $\theta= \pm 110^{\circ}$ ) to minimize cross-talk between left and right channels of a binaural signal, while using the center loudspeaker to cancel any remaining cross-talk. They reported that sounds simulated with this method were accurately perceived, especially for $\phi \geq 40^{\circ}$ in the coronal plane [4].

\section{PROPOSED METHOD}

In this section, the proposed method is described for a general ring of loudspeakers followed by an application for a loudspeaker ring as described in the ITU-R BS775-3 recommendation.

\subsection{General}

The diffuse-field equalization described by Gardner [21] removes non-directional effects (like those caused by microphones, ear canals, etc.) from HRTF recordings. Based on this method, a filter was devised to mitigate the effect of loudspeaker locations on the intended signal at the listener's ears without pursuing cross-talk cancelation.

The number of active loudspeakers varies depending on source elevation in this method: A single one for sources colocated with a loudspeaker; the closest pair that straddle a desired azimuth for sources in the horizon; and as many available at each side of a listener for sources with elevation $\phi \neq 0^{\circ}$. The rationale behind using lateral loudspeaker groups is that sources at the zenith reach the front and back of the pinna simultaneously, whereas sources in the front or back reach that equivalent side of the pinna earlier. Other elevated sources behave somewhere between these two extremes. Reaching simultaneously front and back of the pinna is impossible to achieve with some combinations of loudspeakers in a ring array, for example, when using two loudspeakers in front of a listener. By using lateral loudspeaker grouping (including those in the anterior and posterior sides) it is expected to better approximate the effect of elevated sources. Note that the proposed method would not effective in ring arrangements where loudspeakers are located only in the anterior or posterior side of the listener.

The spatialized version $y$ of a virtual source represented by a monophonic sound $x$ at the location $d$ (with azimuth $\theta$, elevation $\phi$, and at the same distance as the loudspeakers) is achieved either by displaying the sound from a single loudspeaker when the source is colocated with it, or by the weighted mix of horizontal spatialization $S_{\theta}$ (imposing $\phi=0^{\circ}$ ) and vertical spatialization $S_{\phi}$ :

$$
y=|\cos (\phi)| S_{\theta}+|\sin (\phi)| S_{\phi} .
$$

These spatialization weights yield a constant power mix. Although the number of active loudspeakers varies with elevation, $y$ has always the same number of channels as loudspeakers in the ring.

$S_{\theta}$ and $S_{\phi}$ are obtained similarly, so they are represented here by $S_{\circ}$ to ease the explanation: 


$$
\begin{aligned}
& S_{\circ}=x * w f ; \\
& f=\frac{h_{d}}{h_{c \_ \text {min }}} .
\end{aligned}
$$

In Eq. (2), $w$ is a scalar to equalize the RMS of the resulting filter to that of the HRIR at $d\left(h_{d}\right.$ in Eq. (3)); $h_{c_{-} \min }$ is a minimum phase filter version of

$$
h_{c}=\mathscr{F}^{-1}\left(\frac{1}{n} \sum_{i=1}^{n}\left|\mathscr{F}\left(h_{i}\right)\right|^{2}\right)^{0.5},
$$

which is the mean energy spectrum density computed over $n$ HRIRs (denoted by $h_{i}$ ). These HRIRs correspond to the loudspeakers locations used in the spatialization.

Left and right channels of the binaural filter $f$ in Eq. (3) are applied to the channels of $y$ corresponding the closest loudspeakers to the left and right of $d$ in horizontal spatialization. In vertical spatialization, left and right channels of $f$ are independently applied to all channels of $y$ corresponding to loudspeakers on the left and right of the listener, respectively.

Inversion of $h_{c \text { min }}$ in Eq. (3) is performed by imposing a limit of $25 \mathrm{~dB}$ on the magnitude spectrum [21]. Minimum phase filters are obtained by using the Hilbert transformation [22]:

$$
h_{- \text {min }}=\operatorname{Re}\left(\mathscr{F}^{-1}(\exp (\overline{\mathscr{H}[\log (|\mathscr{F}(h)|)]}))\right) .
$$

Finally, only the first 1,024 taps are retained whenever convolution of HRIRs is performed.

\subsection{Application to ITU-R BS.775-3 Layout}

In an ITU-R BS.775-3 layout (loudspeaker elevation $\phi_{L}=0^{\circ}$, and azimuths $\left.\theta_{L}=\left[0^{\circ}, \pm 30^{\circ}, \pm 110^{\circ}\left( \pm 10^{\circ}\right)\right]\right)$, a single loudspeaker is used if the source location is equal to that of one of the loudspeakers as exemplified in the top panel of Fig. 1. Otherwise, when the source elevation $\phi=0^{\circ}$ (in the horizon), two contiguos loudspeakers are used depending on the source azimuth (i.e., either one of these $\theta_{L}$ combinations is used: $\left[0^{\circ}, \pm 30^{\circ}\right],\left[30^{\circ}, 110^{\circ}\right]$, $\left[-30^{\circ},-110^{\circ}\right],\left[110^{\circ},-110^{\circ}\right]$; no vertical spatialization is used in this case as shown in the second panel from the top of Fig. 1. When the source elevation $\phi \neq 0^{\circ}$, besides the horizontal spatialization, vertical spatialization is also carried out: loudspeakers at $\left[30^{\circ}, 110^{\circ}\right]$ are used for displaying the right channel of $f$ and loudspeakers at $\left[-30^{\circ},-110^{\circ}\right]$ for the left channel of the same filter. To each of these two groups, Eq. (2) is applied independently and the final spatialization is the weighted mix of horizontal and vertical spatializations. Depending on the azimuth of the source, four or five loudspeakers (in case $|\theta|<30^{\circ}$ ) are used, as shown in the two bottom panels of Fig. 1.

A Matlab implementation of this method for an ITU-R BS775-3 loudspeaker array configuration is available at

$$
\theta=0^{\circ}, \phi=0^{\circ}, d=160 \mathrm{~cm}
$$
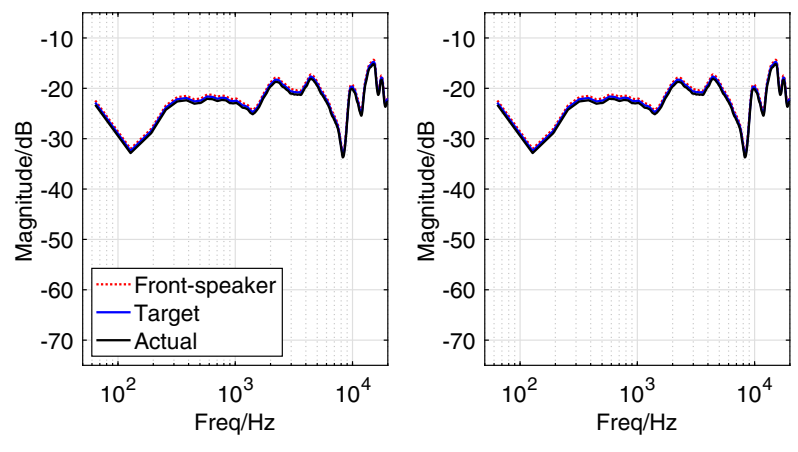

$\theta=70^{\circ}, \phi=0^{\circ}, d=160 \mathrm{~cm}$
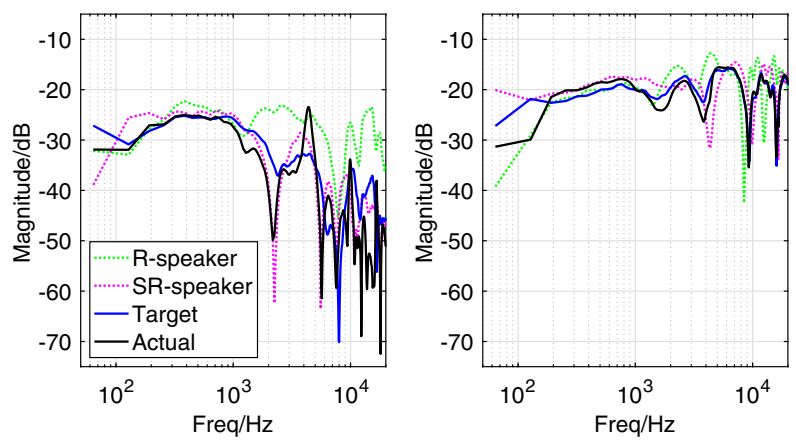

$\theta=90^{\circ}, \phi=60^{\circ}, d=160 \mathrm{~cm}$
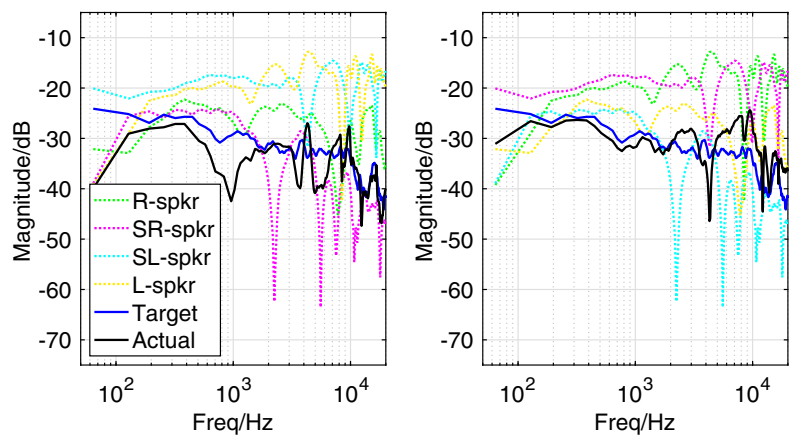

$\theta=20^{\circ}, \phi=60^{\circ}, d=160 \mathrm{~cm}$
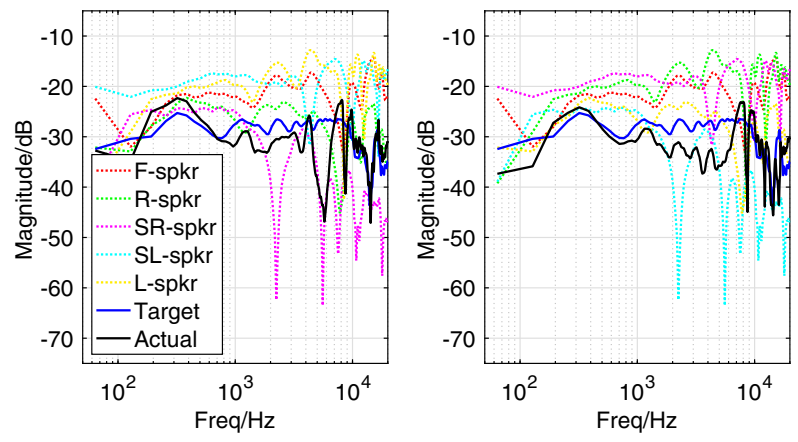

Fig. 1 Examples of target and actual transfer functions for left and right channels (in the respective panels) achieved with the proposed method in an ITU-R BS775-3 loudspeaker array, along with transfer functions of loudspeaker locations used in each case: one, two, four, and five loudspeakers from the top panel. 
http://onkyo.u-aizu.ac.jp/software/eqf/. In this implementation, users may edit the main script to indicate the directory path of a monophonic sound source, the location of the HRIR database, the destination path of the spatialized sounds, the actual location of loudspeakers, and the location of the sound source. Spatialized sounds are stored as Extensible Wave-Format files (six-channel format, corresponding to Left, Right, Center, LF, Surround Left, and Surround Right loudspeakers). Examples of such files are also available from the aforementioned URL.

Note that previous attempts of similar spatialization methods performed in our laboratory were reported in [23-25]. Concretely, we reported in [25] the results of the subjective experiment in echoic conditions also reported in here for completeness. To better assess the accuracy of the proposed method, an objective evaluation and a subjective evaluation in anechoic conditions were performed. They are discussed in the following sections.

\section{OBJECTIVE EVALUATION}

Simulations were carried out to compare the proposed method (Equalizing Filters-EF) with Cross-Talk Cancelation (CTC), and 2D-VBAP (as implemented in [26]). To that end, a loudspeaker array as specified in the ITU-R BS775-3 recommendation was assumed. Since 2D-VBAP cannot reproduce elevated sources, it was only used for horizontal localization comparisons.

HRIRs were retrieved from a diffuse-field equalized version of the database published by Qu et al. [27]. Sound sources were simulated to be at $160 \mathrm{~cm}$ from the center of the head of a listener seated at the center of the array. Elevations $-40^{\circ} \leq \phi \leq 90^{\circ}$, every $10^{\circ}$; and azimuths in the right hemisphere, $0^{\circ} \leq \theta \leq 180^{\circ}$, were used according to the availability of the database: every $5^{\circ}$ for $\phi \leq 50^{\circ}$, and every $10^{\circ}, 15^{\circ}, 30^{\circ}, 360^{\circ}$ for $\phi=60^{\circ}, 70^{\circ}, 80^{\circ}$, and $90^{\circ}$, respectively. Loudspeakers were simulated to be at $\phi_{L}=0^{\circ}$, and $\theta_{L}=\left[0^{\circ}, \pm 30^{\circ}, \pm 110^{\circ}\right]$, at the same distance as the simulated sources.

For the simulation of Cross-Talk Cancelation method, surround loudspeakers $\left( \pm 110^{\circ}\right)$ were selected. Cross-talk for all methods was simulated as explained in [28]. When inversion of HRIRs was necessary, it was performed as explained before, i.e., imposing a limit of $25 \mathrm{~dB}$ on the magnitude spectrum.

To compare the results of different methods, the spectral distortion*

$$
\mathrm{SD}=\left(\frac{1}{n} \sum_{i=2}^{n+1} \epsilon^{2}\right)^{0.5}[\mathrm{~dB}]
$$

in the range of $70 \mathrm{~Hz}-16 \mathrm{kHz}$ was used, i.e., for 1,024-bin

${ }^{*}$ SD should not to be confused with the standard deviation $S D$ (here written in italic typefont).

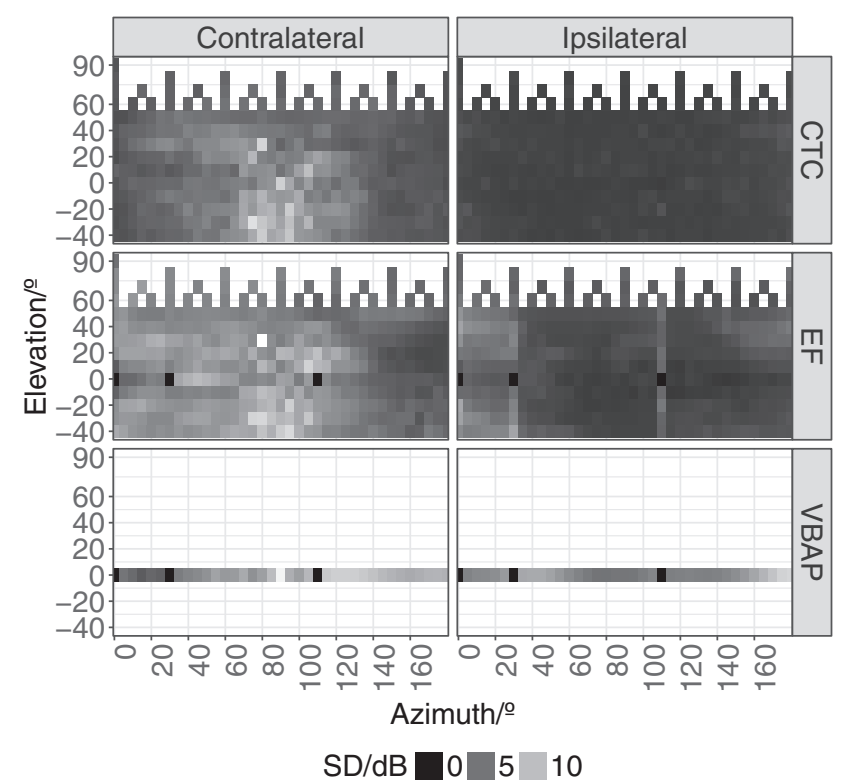

Fig. 2 Spectral distortion (SD) results for Cross-Talk Cancellation (CTC), Equalizing Filter (EF), and 2D-VBAP methods on the right hemisphere.

HRTFs, 249 frequency bins starting from the second one were used. The error $\epsilon$ between the target $(H)$ and achieved $(\hat{H})$ HRTFs was computed as

$$
\epsilon=20 \log \left(\left|H\left(f_{i}\right)\right|\right)-20 \log \left(\left|\hat{H}\left(f_{i}\right)\right|\right) .
$$

\subsection{Results}

Spectral distortion estimations for the three methods are shown in Fig. 2. In the following analyses, Box-Cox transformations of SD were used to address heteroscedasticity in the data, but figures and plots are presented using the actual SD values.

\subsubsection{Horizontal spatialization}

Box-Cox transformed SD were subjected to an ANCOVA controlling for the effect of Azimuth. Method (CTC, EF, VBAP), Ear (ipsilateral, contralateral), and interactions Azimuth-Method and Method-Ear were used as predictors.

The ANCOVA revealed a significant effect on SD of Method $\left[F(2,213)=50.93, p<0.001, \eta_{p}^{2}=0.324\right]$, Ear $\left[F(1,213)=78.03, p<0.001, \eta_{p}^{2}=0.268\right]$, and the interactions Method-Azimuth $[F(2,213)=14.61, p<0.001$, $\left.\eta_{p}^{2}=0.121\right]$, and Method-Ear $[F(2,213)=13.43, p<$ $\left.0.001, \eta_{p}^{2}=0.077\right]$.

On average, VBAP yielded the highest SD $(7.0 \mathrm{~dB}$ cf. $3.57 \mathrm{~dB}$ and $3.32 \mathrm{~dB}$, obtained for EF and CTC, respective1y). Further post-hoc analysis based on Tukey's Honest Significant Difference revealed significant differences between VBAP and CTC $(p<0.001)$, and between VBAP and EF $(p<0.001)$, but not between CTC and EF ( $p=0.999)$. 


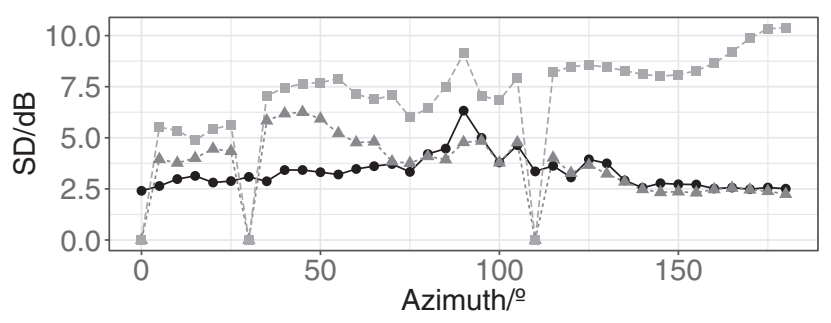

Method $\rightarrow$ CTC - EF - VBAP

Fig. 3 Effect of the interaction between Method and Azimuth on Spectral Distortion (SD).

The mean SD in the contralateral ear was larger than in the ipsilateral ear $(5.95 \mathrm{~dB}$ vs. $3.32 \mathrm{~dB})$. All methods yielded higher SD for the contralateral ear, but whereas the mean SD difference between CTC and EF was small $(<0.5 \mathrm{~dB})$ regardless of ear, the SD difference between VBAP and the other two methods was large: $\sim 4.6 \mathrm{~dB}$ for the ipsilateral ear and $\sim 2.3 \mathrm{~dB}$ for the contralateral ear.

The interaction Method-Azimuth was complex: as expected, no spectral distortion was noted for sources colocated with loudspeakers (in VBAP and EF methods), but some distortion was found in the case of the CTC method. Distortion measures in the back hemisphere were similar (i.e., decreasing) for CTC and EF methods, but not so for VBAP, for which the distortion was greatest, as shown in Fig. 3.

\subsubsection{Elevated spatialization}

An additional ANCOVA controlling for the effect of elevation was performed to compare only CTC and EF methods. Significant effects on the Box-Cox transformed spectral distortion SD were found for factors Method $\left[F(1,1633)=304.71, p<0.001, \eta_{p}^{2}=0.157\right]$ and Ear $\left[F(1,1633)=1900.45, p<0.001, \eta_{p}^{2}=0.538\right] . \quad$ Interactions between Elevation-Method $[F(1,1633)=6.61$, $\left.p=0.010, \eta_{p}^{2}=0.004\right]$, Method-Ear $[F(1,1633)=4.30$, $\left.p=0.038, \eta_{p}^{2}=0.003\right]$, and Elevation-Ear $[F(1,1633)=$ 55.32, $p<0.001, \eta_{p}^{2}=0.032$ ] were also significant.

As in the horizontal case, mean SD for the contralateral ear was larger than that of the ipsilateral ear $(5.5 \mathrm{~dB}$ vs. $2.39 \mathrm{~dB}$ ). Also, as expected, CTC yielded smaller SD than EF $(3.3 \mathrm{~dB}$ vs. $4.6 \mathrm{~dB})$ on average. The mean difference between SD yielded by the two methods was larger for the contralateral ear $(1.39 \mathrm{~dB})$ than for the ipsilateral ear $(1.2 \mathrm{~dB})$, CTC having the lesser distortion in both cases. As shown in Fig. 4, both methods observed a decrease in SD for the contralateral ear as elevation increased, and whereas for CTC the ipsilateral SD was almost flat at $2 \mathrm{~dB}$, it was always higher and increasing with extreme elevations for EF. Grouping the results of the two methods resulted in a similar picture: a decreasing SD with increasing elevation for the contralateral ear and a somewhat flatter SD for the ipsilateral ear, a trough near $\phi=0^{\circ}$ notwithstanding.

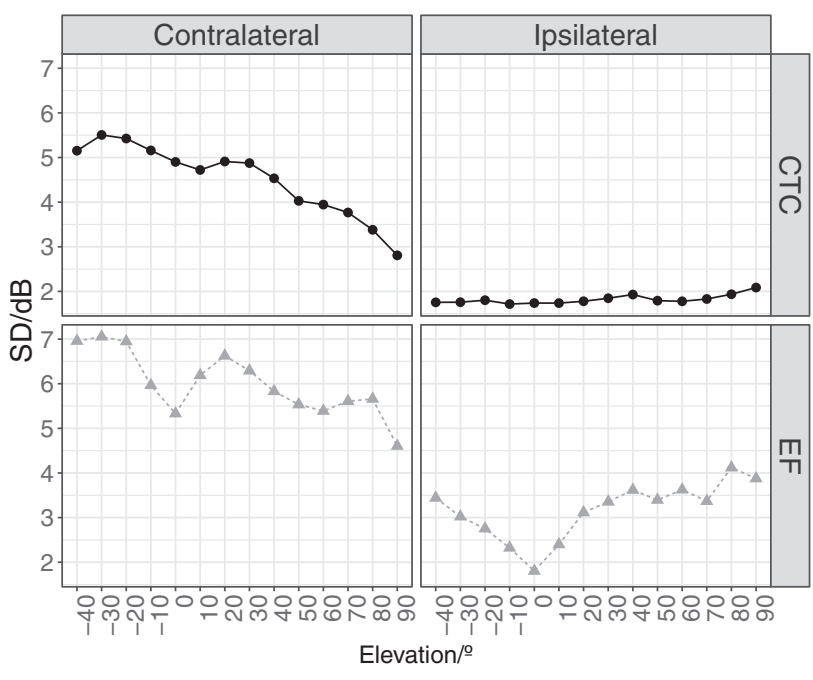

Fig. 4 Effect of the interaction between Method and Ear on SD.

\subsubsection{Interim discussion}

In terms of spectral distortion SD, the proposed method is better than 2D-VBAP in horizontal localization, but inferior to CTC in all cases. Spectral distortion of the proposed method was smaller for the ipsilateral ear in all conditions, which is interesting since the peak and notches of this ear have been found to be more important than those of the contralateral ear for elevation perception, at least for sources rising $\geq 40^{\circ}$ on the mid-sagittal plane [29]. Whether the differences between EF and CTC translate into poor subjective localization accuracy of sources spatialized with the proposed method is investigated in the following section.

\section{SUBJECTIVE EVALUATIONS}

Subjective evaluations were carried out in anechoic and echoic conditions. Descriptions that apply to both experiments are presented first, and differences between the two setups are detailed in Sects. 5.5 and 5.6.

\subsection{Materials}

Monophonic drum riffs sampled at $44.1 \mathrm{kHz} / 24$-bit as the one in Fig. 5 were spatialized to be at a radius of $140 \mathrm{~cm}$ from the center of the loudspeaker array, in the right hemisphere, i.e., at a combination of azimuth $\theta=$ $\left[0^{\circ}, 30^{\circ}, 60^{\circ}, 90^{\circ}, 120^{\circ}, 150^{\circ}, 180^{\circ}\right]$ and elevation $\phi=$ $\left[-30^{\circ}, 0^{\circ}, 30^{\circ}, 60^{\circ}, 90^{\circ}\right]$. Sources at the zenith were evaluated once, reducing the total number of locations to 29 .

\subsection{Apparatus}

Yamaha MSP3 powered loudspeakers were located towards the center of a room, $1.2 \mathrm{~m}$ above the floor, and at a radius of $1.4 \mathrm{~m}$ in an ITU-R BS775-3 layout. Lateral loudspeakers were located at $\pm 30^{\circ}$ and $\pm 120^{\circ}$. The latter 


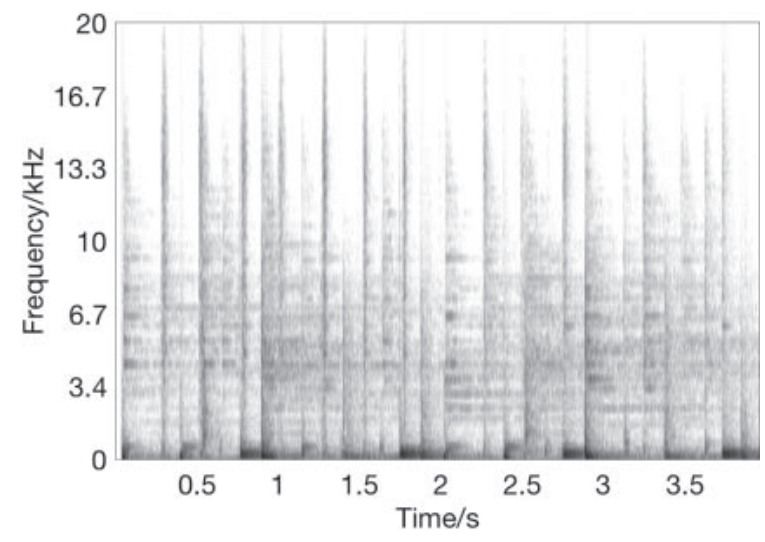

Fig. 5 Spectrogram of a monophonic drum riff recording used in the experiments.

angles were preferred over the traditional $\pm 110^{\circ}$ aperture to reduce the arc covered by the surround loudspeakers.

Tone controls of these loudspeakers were set to their mid-point, and their power was adjusted so that each of them yielded $68 \mathrm{~dB}(\mathrm{~A})$ at the center of the array when pink noise was played back through them. Levels were measured with a Brüel \& Kjær Hand-held Analyzer 2250 Light. Loudspeakers were connected to a computer via an Edirol UA-101 USB interface. The self-paced experiment was programmed using an web survey tool [30] running locally. HRIRs were estimated using barycentric interpolation [31] from a diffuse-field equalized version of the distance-dependent database described in [27].

\subsection{Procedure}

Permission for performing these experiments was obtained following the University of Aizu ethics guidelines. Paid students of the same institution were hearingscreened, considering normal hearing thresholds those that were $\leq 20 \mathrm{~dB}$ (HL) in the range of $0.125-8 \mathrm{kHz}$, as measured with a MAICO MA25 audiometer. Instructions were verbally given following a predetermined script. Assessors sat on a piano bench at the center of the loudspeaker array and their ear heights were visually matched to those of the loudspeakers by adjusting the bench level. Participants were asked to refrain from moving their head or changing posture while listening the stimuli and giving their estimation. This was continuously verified by the experimenter throughout a session. Two demonstrations were offered after blindfolding the assessors for the rest of the experiment. In these demonstrations, a synthesized female voice was presented stating the azimuth and elevation from its intended location. The first demonstration presented all possible elevations (from low to high) for a given azimuth (from front to back); the second demonstration presented all possible azimuths for a given elevation in the same order as the previous demonstration.
Demonstrations preceded a practice session in which oral feedback was provided to 58 location judgements of a $3 \mathrm{~s}$ drum riff. Each target location was rehearsed twice. After the practice session, estimates of the location of a $\sim 4 \mathrm{~s}$ drum riff, spatialized at 29 different locations, were collected from each subject.

The task for the subjects was to orally express the azimuth and elevation of a given stimulus. Opinions were logged in the system by the experimenter. Assessors progressed in the task at their own pace, listening to each stimulus as many times as desired before stating their opinion. On average, each session lasted $\sim 45$ minutes (standard deviation $S D=10.1$ ). Stimuli were randomly permuted per participant within practice and main task sessions.

\subsection{Statistical Analysis}

Horizontal and vertical reversals, angular error, and spherical dispersion computed over opinions (with resolved horizontal confusions) are reported. Angular error $\omega$ for each opinion was computed as

$$
\omega=\cos ^{-1}\left(\frac{\boldsymbol{d}_{t} \cdot \boldsymbol{d}_{\boldsymbol{r}}}{\left|\boldsymbol{d}_{\boldsymbol{t}}\right|\left|\boldsymbol{d}_{\boldsymbol{r}}\right|}\right)
$$

where $\boldsymbol{d}_{t}$ and $\boldsymbol{d}_{\boldsymbol{r}}$ are vectors with the same direction as the target and reported location, respectively. Angular error comprises errors in the judgement of azimuth and elevation as well as the effects of response variability and systematic response bias [32].

The inverse of the concentration parameter $\kappa$ in the von Mises-Fisher distribution was used as a measure of spherical dispersion. It was approximated as

$$
\hat{\kappa}^{-1}=\frac{1-\bar{r}^{2}}{3 \bar{r}-\bar{r}^{3}} .
$$

where $\bar{r}$, the normalized length of a vector considering all the opinions for a target location, is computed as

$$
\bar{r}=\frac{\left|\sum_{i=1}^{n} \boldsymbol{d}_{\boldsymbol{r} i}\right|}{n} .
$$

In Eq. (10), $\boldsymbol{d}_{\boldsymbol{r} i}$ is a unit vector corresponding to the reported location of the $i$-th assessor, and $n$ is the number of assessors [33]. Small values of $\hat{\kappa}^{-1}$ indicate more tightly concentrated estimates.

\subsection{Anechoic Conditions}

The anechoic chamber at the University of Aizu was used in this experiment. This room $(5 \mathrm{~m}$ wide, $6 \mathrm{~m}$ long, $5 \mathrm{~m}$ high) has an mean $\mathrm{RT}_{60}=67 \mathrm{~ms}$, Noise Criterion $\mathrm{NC}=18[8 \mathrm{kHz}]$, and Room Criteria Mark II RC $=15$ (HF) with Quality Assurance Index QAI $=8.4 .25$ males and 3 females with an average age of 22 years $(S D=2.4)$ 
participated in this experiment. Additional data collected from 2 male participants were excluded since they had hearing thresholds $>20 \mathrm{~dB}$ (HL) in more than one of the inspected bands.

\subsection{Echoic Conditions}

The experiment was conducted in a regular room ( $7.5 \mathrm{~m}$ wide, $7 \mathrm{~m}$ long, $2.7 \mathrm{~m}$ high) with mean $\mathrm{RT}_{60}=$ $322 \mathrm{~ms}, \mathrm{NC}=25$ [500], and $\mathrm{RC}=26(\mathrm{~N})$ with $\mathrm{QAI}=2.6$.

25 males and 5 females with a mean age of 21 years $(S D=1.47)$ participated in this experiment. Because of a configuration error, loudspeakers in this experiment were calibrated by playing back white noise (instead of pink) so that at the center of the loudspeaker array the pressure level was $65 \mathrm{~dB}$ (A). This error, however, did not seem to affect the results, as described below.

\section{RESULTS}

Collected responses are shown in Fig. 6. In this plot, assessors are represented by a shape according to performance: upward triangles represent assessors for whom mean angular error was within the first quartile $\left(24.9^{\circ} \leq \omega<\right.$ $\left.35.8^{\circ}\right)$, downward triangles, those within the fourth quartile $\left(42.6^{\circ}<\omega \leq 56.2^{\circ}\right)$, and squares for the rest.

\subsection{Spherical Dispersion}

As shown in Fig. 6, spherical dispersion was similar for all combinations of target azimuth $\theta$ and elevation $\phi$, smaller values at $\left(\theta=0^{\circ}, \phi=0^{\circ}\right)$ and $\left(\theta=120^{\circ}, \phi=0^{\circ}\right)$ notwithstanding. The smallest dispersion was found for $\kappa^{-1}\left(0^{\circ}, 0^{\circ}\right)=0.019$ and the largest for $\kappa^{-1}\left(150^{\circ},-30^{\circ}\right)=$ 0.030. An ANOVA revealed no significant effect of azimuth $(p=0.110)$ or elevation $(p=0.160)$ on spherical dispersion.

\subsection{Reversals}

Opinions were regarded as reversals when their absolute value was equal to the target location, but the
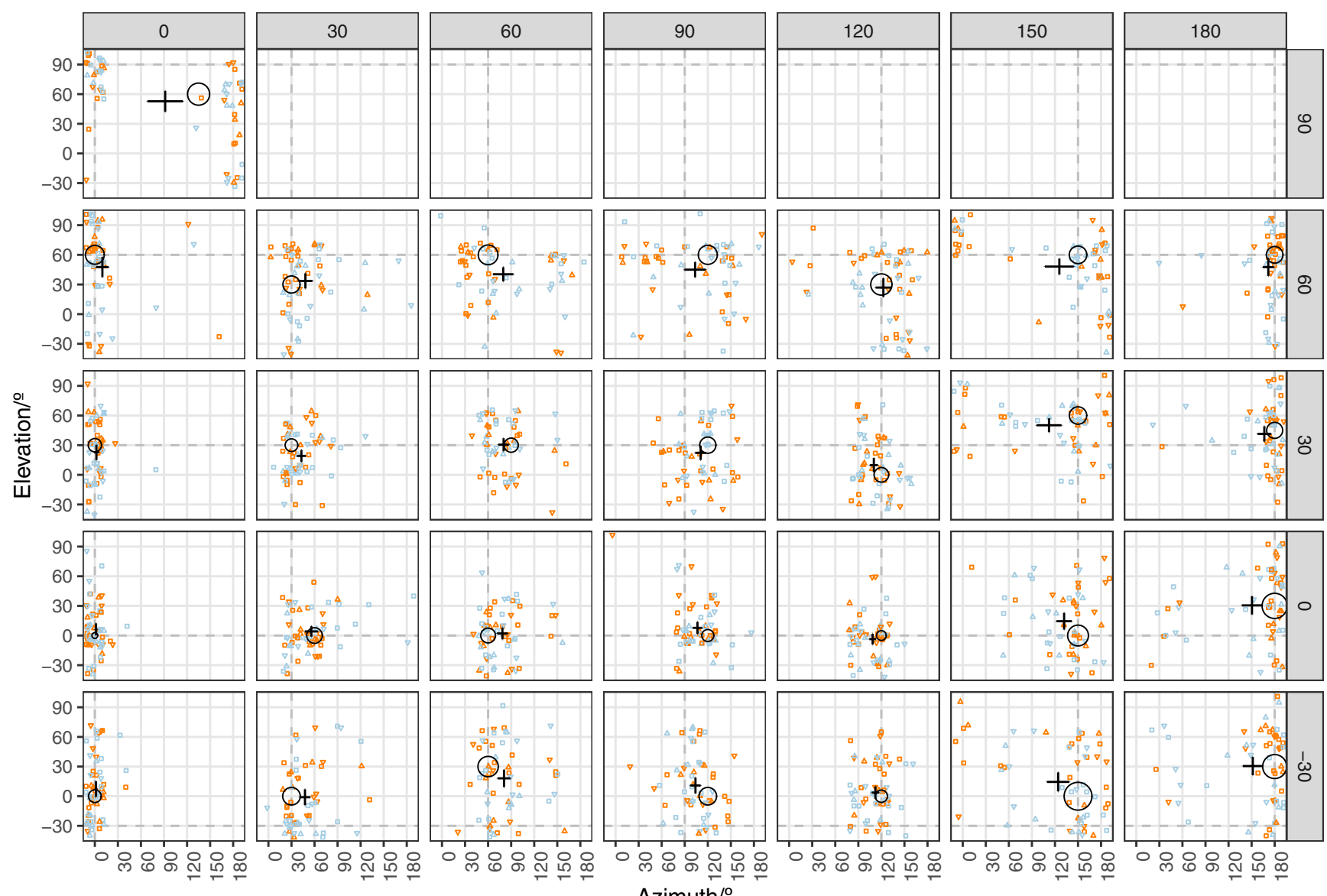

Azimuth/o

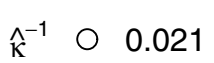

0.024

0.027

0.030

Anechoic

Echoic

Fig. 6 Target locations (in panels) vs. reported ones after horizontal confusion corrections. Assessors are represented by shapes: upward and downward triangles for good and bad classifiers, and squares for the rest (see the text for a detailed explanation). Less than $15^{\circ}$ of jitter in each direction was added to each opinion to ease visual inspection. Circles are centered at the median opinion and their sizes correspond to the spherical dispersion. Error bars, centered at the mean opinion, indicate $95 \%$ CIs; dashed lines intersect at the target location of each panel. 


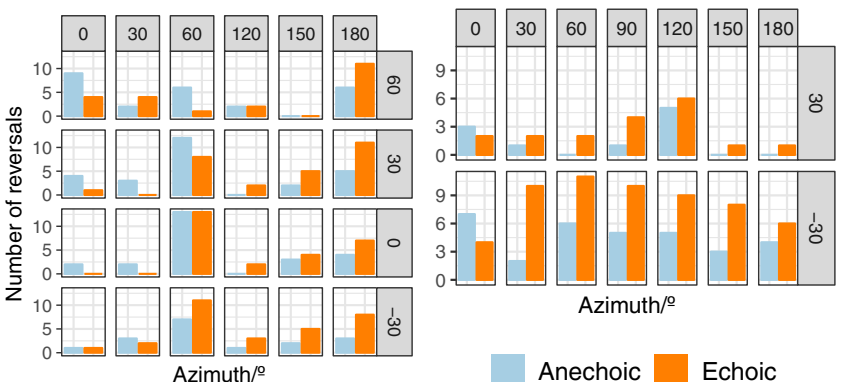

Fig. 7 Horizontal reversals (left) and vertical ones (right).

signs between target and reported angle differed. These are presented in Fig. 7. Reversals percentages are computed over the total number of judgements where reversals were possible (i.e., excluding judgements at $\theta=90^{\circ}$, and $\phi=$ $\left.\left[0^{\circ}, 60^{\circ}, 90^{\circ}\right]\right)$.

Reversals were more common in echoic than anechoic conditions: $30.2 \%$ vs. $26.4 \%$ in horizontal judgements, and $65.5 \%$ vs. $36.2 \%$ in vertical ones. Regardless of room, mistaking a negative elevation for a positive one was about three times as common as the opposite case $(\sim 76 \%$ vs. $\sim 24 \%$ ). Whereas front to back reversals were more common in anechoic conditions ( $\sim 70 \%$ vs. $\sim 30 \%$ ), back to front confusions dominated in echoic conditions ( $\sim 57.1 \%$ vs. $\sim 42.9 \%)$.

Assuming a bitragion breadth of $14.5 \mathrm{~cm}$, the surround loudspeaker was about $2 \mathrm{~cm}$ closer to the ipsilateral ear than the front loudspeaker. This discrepancy suggests that precedence effect could explain some of the confusions: e.g., there were more confusions for $\theta=60^{\circ}$ where there was no loudspeaker than for $\theta=120^{\circ}$ where the loudspeaker was located.

\subsection{Angular Errors}

A mixed-ANOVA with Room (Anechoic, Echoic) as between-subject factor, and target Azimuth and Elevation (excluding $\phi=90^{\circ}$ since azimuth at that elevation is irrelevant) as within-subject factors was performed to investigate their effect on angular error. Sphericity assumptions were violated in all cases (as confirmed with
Mauchly's tests, $p<0.002$ ), so degrees of freedom and p-values were corrected using Greenhouse-Geisser $\epsilon$ estimates of sphericity.

This ANOVA revealed significant effects of Elevation $\left[F(2.34,131.21)=34.1, p<0.001, \eta_{g}^{2}=0.007\right]$, and Azimuth $\quad\left[F(4.72,264.10)=14.72, p<0.001, \eta_{g}^{2}=\right.$ $0.006]$. The effect of Room was not significant $[F(1,56)=$ $\left.0.05, p=0.827, \eta_{g}^{2}<0.001\right]$. The interaction between Elevation and Azimuth was significant $[F(11.7,653.2)=$ $\left.6.69, p<0.001, \eta_{g}^{2}=0.067\right]$, and so were the interactions between Room and Azimuth $[F(4.72,264.10)=3.04$, $\left.p=0.013, \eta_{g}^{2}=0.012\right]$, and Room and Elevation $\left[F(2.34,131.21)=3.54, p=0.025, \eta_{g}^{2}=0.008\right]$.

As shown in the left panel of Fig. 8, larger angular errors were found for negative elevations $(p<0.001)$; no significant differences between other elevations were found when the effect of room was not considered. This was confirmed with a post-hoc analysis based on t-tests with Bonferroni corrections. A similar analysis revealed significant differences between front $\left(\theta=\left[0^{\circ}, 30^{\circ}\right]\right)$, side $\left(\theta=\left[30^{\circ}, 60^{\circ}, 90^{\circ}, 120^{\circ}\right]\right)$, and back azimuths $(\theta=$ $\left.\left[150^{\circ}, 180^{\circ}\right]\right)$, but not among azimuths within each of these groups, as illustrated in the center panel of Fig. 8.

Regardless of room, a similar trend in the mean angular error was found for Elevation and Azimuth, but results of the echoic room indicated significantly larger error for target elevation $\phi=-30^{\circ}(p=0.010)$, and target azimuth $\theta=150^{\circ}(p=0.003)$, as shown in the left and center panels of Fig. 8. It is possible that the loudspeaker locations became more conspicuous in the echoic room than in anechoic conditions. Spreading the signal between loudspeakers as commonly done in VBAP implementations could help to prevent this issue.

The interaction Azimuth-Elevation was complex, as shown in the right panel of Fig. 8. Largest and smallest errors were produced by target elevation $\phi=-30^{\circ}$ and $\phi=0^{\circ}$, respectively. Mean error differences among elevations for a given azimuth were smaller when targets were colocated with loudspeakers $\left(10.3^{\circ}, 19.0^{\circ}, 21.6^{\circ}\right.$ for $\theta=$ $30^{\circ}, 120^{\circ}, 0^{\circ}$ ); the largest mean difference was found for target azimuth $\theta=180^{\circ}$.
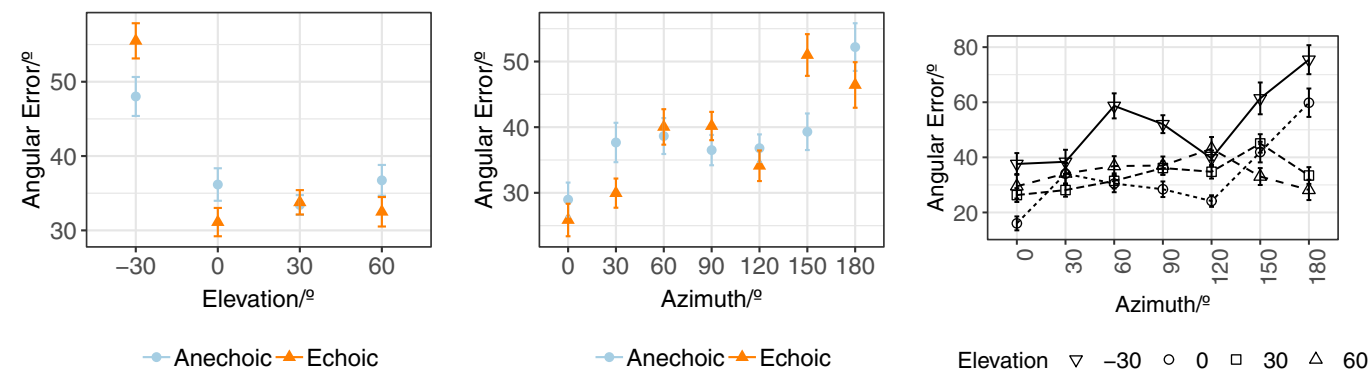

Fig. 8 Effects on angular error of the interactions between room and target elevation (left), room and target azimuth (center), and azimuth and elevation (right). Error bars indicate 95\% CIs. 
As shown in Fig. 6, results for target locations in the mid-sagittal plane are similar to the trend found by Damaske and Wagener [34] (as cited in [35]). That is, subjective responses presented here were biased towards the front, and angular error increased in locations at the back. Angular errors in targets near the coronal plane $\left(\theta=\left[60^{\circ}, 90^{\circ}, 120^{\circ}\right]\right)$ displayed a convex pattern whereby angular error increased as absolute elevation increased, a trend similar to that reported by Ashby et al. when using real sources [36].

\section{DISCUSSION}

\subsection{Comparison with Other Methods}

Results of objective comparisons indicate that spectral distortion of the proposed method is greater than that of CTC but less than that of VBAP (for horizontal spatializations). Direct subjective comparisons with other methods are beyond the scope of this manuscript, but comparisons with mean angular errors and other metrics reported elsewhere are offered in lieu of that. These comparisons illustrates somehow the performance of the proposed method, but should not be taken as a way of ranking it.

Mean angular error for all directions in the experiments reported here was $\omega=38.4^{\circ}$ with a mean spherical dispersion $\kappa^{-1}=0.022$. These figures are comparable to those reported by Gardner [21] for СТC stimuli $\left(\omega=32.4^{\circ}\right.$, $\kappa^{-1}=0.130$ ); but subpar compared to those found for short stimuli presented via headphones with individualized HRTFS $\left(\omega=16.2^{\circ}-21.1^{\circ}, \kappa^{-1}=0.013-0.127\right)$ [37], and those found for bursts of noise presented via loudspeaker in anechoic conditions $\left(\omega=16.1^{\circ}-29.8^{\circ}, \kappa^{-1}=0.030\right.$ 0.070) [38].

Kim et al. [20] did not report angular errors, thus a comparison on the mean elevation response range is presented instead: In their experiments, responses ranged between $20^{\circ}$ and $50^{\circ}$ (approximately) for broadband noise artificially elevated to be at $\phi=\left[30^{\circ}, 50^{\circ}, 70^{\circ}\right]$ in the coronal plane. This range was $\left[11^{\circ}, 45^{\circ}\right]$ in the experiments discussed in Sect. 5 for elevation $\phi \leq 60^{\circ}$ in the same plane. I.e., compressions in the response range were similar for both methods: $66.7 \%$ and $62.2 \%$, respectively.

\subsection{Compression in the Elevation Response Range}

In contrast with elevation judgements of sources spatialized to be below the listeners, sources spatialized to be above them were, with some accuracy, correctly identified. Interestingly, a range compression in elevation responses was observed: whereas the target range was $\left[-30^{\circ}, 90^{\circ}\right]$, the mean reported range was $\left[-3.6^{\circ}, 53^{\circ}\right]$, as shown in Fig. 6.

Reasons for such vertical shift and range compression are still unknown. However, they have been observed in other studies: Kim et al. [20] reported a leveling in subjective responses to real and virtual sources, regardless of the experimental apparatus used (loudspeakers or headphones); some of the data provided by Wightman \& Kistler [38] observe a similar trend; and more recently, this pattern was observed in data collected by Ferguson \& Cabrera [39].

Hartmann et al. [40] have associated similar elevationdependent degradation with temporal and intensity aspects of the signal. Authors in [39] suggest a possible ceiling/ flooring statistical effect in the computation of averages, whereas Kim et al. suggested that this range compression was associated with discrepancies between assessors' HRTFs and the generic ones used for their spatialization: As a sound source moves from low to high elevations, notches above $5 \mathrm{kHz}$ (related to pinna reflections) traverse the spectrum from low to high [9]. The actual location of these notches is highly dependent on the listener: larger pinnae yield lower notch center frequencies and vice versa [41]. When listeners with pinnae larger than those used to capture an HRTF are subjected to sound spatialized with that HRTF, there is an upward shift on their estimation of that sound's elevation [42]. This elevation shift is reversed for listeners with pinnae smaller than those used in the capture of HRTFs. Mismatches in pinna size could explain elevation judgement degradation for virtual sources, but they do not explain a similar trend observed for real sources in [20] and [38].

Recently, Clarke \& Lee [43] reported that by artificially reducing the magnitude of spectral notches related to pinna reflections, the location of a sound is shifted upwards when it lays on the mid-sagittal plane. They follow up the research of Macpherson and Sabin [44] who studied this elevation judgement degradation at several azimuths and different spectral manipulations. The latter study found that as notches or peaks were progressively leveled, elevation judgement degraded in a similar fashion as described before.

To summarize, on the one hand, changing the center frequency of spectral notches associated with pinna reflections produces vertical shifts in elevation judgements; on the other hand, decreasing the magnitude of spectral peaks and troughs seems level slopes in perceived vs. target elevation plots. So, upward shift and range compression of reported elevations in the experiments here conducted could have several explanations:

i) Stimuli at $\phi=90^{\circ}$ was presented less often than stimuli at $\phi=60^{\circ}$ (in fact, it was only presented once in the main task); due to the lack of contrast opportunities, it is possible that some subjects ascribed high elevations to the $\phi=60^{\circ}$ condition.

ii) Pinna size mismatches and changes on the magnitude of spectral peaks and notches (as byproduct of loudspeaker locations and their frequency responses). 
Although assessors' pinnae were not measured, it is reported in [27] that a "small pinna" simulator was placed on the right ear of the manikin used to capture the HRTF database, so presumably this simulator was as big or smaller than the pinnae of most participants.

iii) Presence of the floor could be influencing vertical judgements, especially in negative elevations: besides the direct path between the sound source and the ears of a listener, reflections on the floor are present in most practical situations. Coloration produced by these reflections increased subjective elevation accuracy for speechlike signals $[45,46]$. In the anechoic condition experiment, such reflections are nonexistent since they are not captured in the HRTFs used; in the echoic condition experiment, these reflections are dominated by the height of the loudspeakers and the head of the listener. Coloration related to floor reflections different from that normally produced by the location of the simulated source could be detrimental to elevation estimations.

\subsection{Limitations of the Study}

In the present study, there were no attempts to control the magnitude of peaks and notches of the processed signals, the influence of loudspeaker impulse responses (the MSP3, a two-way loudspeaker, has a rather uneven response above $5 \mathrm{kHz}$ ), or pinna size differences between those used to capture the HRTF database and those of the subjects. In the same vein, phase effects produced by minute differences in the distance between the loudspeakers and a subject's ears were not corrected: as previously mentioned, surround loudspeakers were $\sim 2 \mathrm{~cm}$ closer than front loudspeakers in the experimental setup, which creates a comb-filter with the first notch at $\sim 8.6$ $\mathrm{kHz}$. It is likely that addressing these issues could improve accuracy of the resulting spatialization. Additionally, subjective comparisons with other spatialization methods are not reported here but planned for future publication.

\section{CONCLUSIONS}

A spatialization method based on diffuse-field equalization and side loudspeaker grouping was proposed as an alternative to display elevation in a ring of loudspeakers. Objective evaluations revealed that this method is better (in terms of spectral distortion) than 2D-VBAP when only horizontal spatialization is considered, but worse than cross-talk cancellation for vertical and horizontal spatialization. Spectral distortion deterioration observed on this method with respect to cross-talk cancelation did not translate into poor subjective performance (measured as angular error), at least for positive elevations.

The proposed method seems to produce adequate sound imagery in echoic and anechoic environments, but further subjective evaluations are needed to assess the perform- ance of the proposed method relative to other spatialization methods. A compression in the reported elevation range described in other research was also found here. Several possible causes were discussed, including the effect of floor reflections some times neglected in spatialization of virtual sources.

\section{ACKNOWLEDGMENTS}

This work was supported by JSPS Kakenhi Grant No. 16K00277. The author express his gratitude to Prof. Michael Cohen and the anonymous reviewers of this manuscript for their invaluable comments and suggestions. He also thanks Mr. N. Fukasawa and Ms. Y. Suzuki for their support on the administration of the experiments.

\section{REFERENCES}

[1] Int. Telecommunication Union, Recommendation ITU-R BS.775-3, Multichannel Stereophonic Sound System with and without Accompanying Picture (2012).

[2] Int. Telecommunication Union, Report ITU-R BS.2159-7 Multichannel sound technology in home and broadcasting applications (2015).

[3] J. Bauck and D. H. Cooper, "Generalized transaural stereo and applications," J. Audio Eng. Soc., 44, 683-705 (1996).

[4] S. Kim, M. Ikeda, A. Takahashi, Y. Ono and W. L. Martens, "Virtual ceiling speaker: Elevating auditory imagery in a 5channel reproduction," Proc. 127 Audio Eng. Soc. Conv., (2009).

[5] H. Jo, W. L. Martens, Y. Park and S. Kim, "Confirming the perception of virtual source elevation effects created using 5.1 channel surround sound playback," Proc. 9th ACM SIGGRAPH Conf. Virtual-Reality Continuum and Its Applications in Industry, pp. 103-110 (2010).

[6] J. Blauert, Spatial Hearing: The Psychophysics of Human Sound Localization (MIT Press, Cambridge, Mass., 1997).

[7] M. Morimoto and H. Aokata, "Localization cues of sound sources in the upper hemisphere," J. Acoust. Soc. Jpn. (E), 5, 165-173 (1984).

[8] J. Hebrank and D. Wright, "Spectral cues used in the localization of sound sources on the median plane," J. Acoust. Soc. Am., 56, 1829-1834 (1974).

[9] P. J. Bloom, "Creating source elevation illusions by spectral manipulation," J. Audio Eng. Soc., 25, 560-565 (1977).

[10] H. Lee, "Phantom image elevation explained," Proc. 141st Audio Eng. Soc. Conv., \#9644 (2016).

[11] C. J. Chun, H. K. Kim, S. H. Choi, S.-J. Jang and S.-P. Lee, "Sound source elevation using spectral notch filtering and directional band boosting in stereo loudspeaker reproduction," IEEE Trans. Consum. Electron., 57, 1915-1920 (2011).

[12] J. Blauert and R. Rabenstein, "Providing surround sound with loudspeakers: A synopsis of current methods," Arch. Acoust., 37, 5-18 (2012).

[13] J. J. Lopez, M. Cobos and B. Pueo, "Rear and side reproduction of elevated sources in wave-field synthesis," Proc. 17th IEEE European Signal Processing Conf. (Eurasip), pp. 804-808 (2009).

[14] J. J. Lopez, M. Cobos and B. Pueo, "Elevation in wave-field synthesis using HRTF cues," Acta Acust. united Ac., 96, 340350 (2010).

[15] W. Jin, M. Marković and L. Pang, "Multizone soundfield reproduction with virtual elevations using a linear loudspeaker 


\section{J. VILLEGAS: ELEVATION PERCEPTION IN LOUDSPEAKERS ARRANGED IN A RING}

array," Proc. 140th Audio Eng. Soc. Conv., \#9575 (2016).

[16] V. Pulkki, "Virtual sound source positioning using vector base amplitude panning,” J. Audio Eng. Soc., 45, 456-466 (1997).

[17] V. Pulkki, M. Karjalainen and V. Välimäki, "Localization, coloration, and enhancement of amplitude-panned virtual sources," Proc. 16th Audio Eng. Soc. Int. Conf., \#16-024 (1999).

[18] V. Pulkki, "Compensating displacement of amplitude-panned virtual sources," Proc. 22nd Audio Eng. Soc. Int. Conf., \#000244 (2002).

[19] L. Mikko-Ville, J. Vilkamo, K. Jussila, A. Politis and V. Pulkki, "Gain normalization in amplitude panning as a function of frequency and room reverberance," Proc. 55th Audio Eng. Soc. Int. Conf., \#3-5 (2014).

[20] S. Kim, M. Ikeda, Y. Ono and A. Takahashi, "Investigating listeners' localization of virtually elevated sound sources," Proc. 40th Int. Audio Eng. Soc. Conf., \#8-5 (2010).

[21] W. G. Gardner, 3-D Audio Using Loudspeakers (Springer Science \& Business Media, US, 1998).

[22] M. O. Hawksford, "Digital signal processing tools for loudspeaker evaluation and discrete-time crossover design," $J$. Audio Eng. Soc., 45(1/2), 37-62 (1997).

[23] S. Nogami, T. Nagasaka, J. Villegas and J. Huang, "Influence of spectral energy distribution on subjective azimuth judgements," Proc. 139th Audio Eng. Soc. Int. Conv., \#9426 (2015).

[24] T. Nagasaka, S. Nogami, J. Villegas and J. Huang, "Influence of spectral energy distribution on elevation judgments," Proc. 139th Audio Eng. Soc. Int. Conv., \#9425 (2015).

[25] J. Villegas, N. Fukasawa and Y. Suzuki, "Improving elevation perception in single-layer loudspeaker array display using equalizing filters and lateral grouping," Proc. 143rd Audio Eng. Soc. Int. Conv., \#9868 (2017).

[26] A. Politis, Microphone array processing for parametric spatial audio techniques, Ph.D. thesis, Aalto University (2016).

[27] T. Qu, Z. Xiao, M. Gong, Y. Huang, X. Li and X. Wu, "Distance-dependent head-related transfer functions measured with high spatial resolution using a spark gap," IEEE Trans. Audio Speech Lang. Process., 17, 1124-1132 (2009).

[28] W. G. Gardner, "Transaural 3-D audio," Technical Report 342, MIT Media Laboratory, Perceptual Computing Section (1995).

[29] E. A. Macpherson and A. T. Sabin, "Binaural weighting of monaural spectral cues for sound localization," J. Acoust. Soc. Am., 121, 3677-3688 (2007).

[30] C. Schmitz, LimeSurvey: An Open Source survey tool, LimeSurvey Project, Hamburg, Germany (2016). URL http:// www . limesurvey . org.

[31] H. Gamper, "Head-related transfer function interpolation in azimuth, elevation, and distance," J. Acoust. Soc. Am., 134, EL547-EL553 (2013).

[32] D. S. Brungart, N. I. Durlach and W. M. Rabinowitz, "Auditory localization of nearby sources. ii. Localization of a broadband source," J. Acoust. Soc. Am., 106, 1956-1968
(1999).

[33] A. Banerjee, I. S. Dhillon, J. Ghosh and S. Sra, "Clustering on the unit hypersphere using von Mises-Fisher distributions," J. Mach. Learn. Res., 6, 1345-1382 (2005).

[34] P. Damaske and B. Wagener, "Directional hearing tests by the aid of a dummy head," Acta Acust. united Ac., 21, 30-35 (1969).

[35] V. Pulkki and M. Karjalainen, Communication Acoustics: An Introduction to Speech, Audio and Psychoacoustics (John Wiley \& Sons, UK, 2015).

[36] T. Ashby, R. Mason and T. Brookes, "Elevation localization response accuracy on vertical planes of differing azimuth," Proc. 136th Audio Eng. Soc. Int. Conv., (2014).

[37] T. Djelani, C. Pörschmann, J. Sahrhage and J. Blauert, “An interactive virtual-environment generator for psychoacoustic research ii: Collection of head-related impulse responses and evaluation of auditory localization," Acta Acust. united Ac., 86, 1046-1053 (2000).

[38] F. L. Wightman and D. J. Kistler, "Headphone simulation of free-field listening. ii: Psychophysical validation," J. Acoust. Soc. Am., 85, 868-878 (1989).

[39] S. Ferguson and D. Cabrera, "Vertical localization of sound from multiway loudspeakers," J. Audio Eng. Soc., 53, 163-173 (2005).

[40] W. M. Hartmann, V. Best, J. Leung and S. Carlile, "Phase effects on the perceived elevation of complex tones," $J$. Acoust. Soc. Am., 127, 3060-3072 (2010).

[41] S. Mehrgardt and V. Mellert, "Transformation characteristics of the external human ear," J. Acoust. Soc. Am., 61, 1567-1576 (1977).

[42] W. L. Martens, "Perceptual evaluation of filters controlling source direction: Customized and generalized HRTFs for binaural synthesis," Acoust. Sci. \& Tech., 24, 220-232 (2003).

[43] J. R. Clarke and H. Lee, "The effects of decreasing the magnitude of elevation-dependent notches in HRTFs on median plane localization," Interactive Audio Systems Symp. (2016).

[44] E. A. Macpherson and A. T. Sabin, "Vertical-plane sound localization with distorted spectral cues," Hear. Res., 306, 7692 (2013).

[45] R. Guski, "Auditory localization: Effects of reflecting surfaces," Perception, 19, 819-830 (1990).

[46] F. Wendt, R. Höldrich, Robertldrich and M. Frank, "The influence of the floor reflection on the perception of sound elevation," Proc. Fortschritte der Akustik-DAGA (2017).

Julián Villegas is an Associate Professor at the Computer Arts Laboratory of the University of Aizu in Japan. His research interests include speech intelligibility, music and sound, psychoacoustics, and spatial sound. He received an Sc.B. in Electronic Engineering from University of Valle (Colombia), M.Sc. and Ph.D. in Computer Sciences from the University of Aizu (Japan). 